\title{
Erratum to: Real-time error compensation of a three-axis machine tool using a laser tracker
}

\author{
Zheng Wang • Paul G. Maropoulos
}

Published online: 10 July 2013

(C) Springer-Verlag London 2013

Erratum to: Int J Adv Manuf Technol

DOI 10.1007/s00170-013-5019-5

The original version of this article, unfortunately, contained an error.

Paul G. Maropoulos's surname was incorrectly spelled as Maropolous. The correct spelling is presented in this paper.

The online version of the original article can be found at http://dx.doi.org/ 10.1007/s00170-013-5019-5.

Z. Wang $(\bowtie) \cdot$ P. G. Maropoulos

Mechanical Engineering, University of Bath, Bath BA2 7AY, UK

e-mail: zw215@bath.ac.uk

P. G. Maropoulos

e-mail: p.g.maropoulos@bath.ac.uk 